\title{
Preparation of PLLA-co-bis A ER/VMT Nanocomposites by In Situ Polymerization Process
}

\author{
Hong $\mathrm{Xu}^{*}$, Yali Bai and Zhiping Mao*
}

\author{
Key Laboratory of Science \& Technology of Eco-Textile (Donghua University), Ministry of Education, Shanghai \\ 201620, China \\ College of Chemistry, Chemical Engineering and Biotechnology, Donghua University, Shanghai 201620, China
}

\begin{abstract}
Poly(L-lactic acid)-co-bisphenol-A epoxy resin/vermiculite nanocomposites (PLLA-co-bis A ER/VMT) were prepared by in situ melt polycondensation of L-LA in the presence of amino-modified vermiculite. The fourier transform infrared (FTIR) spectra was used to investigate molecular interactions between the modified vermiculite and PLLA. Transmission electron microscopy (TEM) testing indicated that the VMT particles were finely dispersed in the PLLA-coBis A ER matrix. The molecular weight kept almost constant at low VMT content $(0.5 \mathrm{wt} \%, 1 \mathrm{wt} \%)$ but decreased at higher VMT content (2\%). The detailed thermal property and crystallization behavior of samples were studied by using polarized optical microscopy (POM), differential scanning calorimetry (DSC) and thermogravimetric analyzer (TGA). The results indicated that the thermal stability, overall crystallization rate and spherulitic texture of PLLA-co-bis A ER were strongly influenced in the presence of vermiculite particles.
\end{abstract}

Keywords: Poly(L-lactic acid)-co-bisphenol-A epoxy resin, vermiculite, nanocomposites, crystallization, thermal property.

\section{INTRODUCTION}

During the last decade, significant attention has been focused on biodegradable polymers. Among all these polymers, poly(lactic acid) (PLA) is one of the most promising because it is thermoplastic, biocompatible and has a high strength, a high modulus, and good processability $[1,2]$. However, PLA also has been revealed an inefficient crystallization process for both the lower crystallization rate and crystallinity as compared with other polymers $[3,4]$.

In recent years, various kinds of layered silicates such as montmorillonite, talc, kaolin, and so on are employed to prepare PLA/OMLS (organically modified layered silicate) for enhancing the performance of PLA [5]. Vermiculite (VMT) like the well-known montmorillonite is a mica-type silicate and possesses a layered structure. It will have a dramatic expansion when subjected to high temperature roasting and obtain excellent heat insulation properties. Because of the unique morphology and structure of VMT, it had been studied for the preparation of composites [6].

In this study, PLLA-co-bis A ER/VMT were prepared by in situ polycondensation. The effect of organo-modified vermiculite on molecular weight, thermal stability and crystallization behavior of the nanocomposite behavior were investigated.

\section{EXPERIMENTAL}

\subsection{Materials}

L-Lactic acid (L-LA) was purchased from Jiangxi Musashino Bio-chem. Co. Ltd. (China) as a $90 \mathrm{wt} \%$ aqueous

*Address correspondence to these authors at the College of Chemistry, Chemical Engineering and Biotechnology, Donghua University, Shanghai 201620, China; Tel: +86-21-6779272; Fax: +86-21-67792707;

E-mails: zhpmao@dhu.edu.cn,hxu@dhu.edu.cn solution of the monomer, $99 \%$ optically pure according to the manufacturer. The native vermiculite was supplied by Zhongyan Building Material Company (China). Bisphenol-A epoxy resin with epoxy equivalent weight of 188 was supplied by Huntsman Advanced Materials (China). Tin II chloride dihydrate $\left(\mathrm{SnCl}_{2} \cdot 2 \mathrm{H}_{2} \mathrm{O}\right)$, adipic acid and THF were purchased from China Medicine (Group). All materials above were analytical grade.

\subsection{Sample Preparation}

The nanometer-sized exfoliated vermiculite was modified with $\gamma$-Aminopropyltriethoxysilane (KH-550) before polymerization. Then the modified-vermiculite was added to lactic acid matrix and mixed thoroughly by a Ultrasonic Cell Crusher. The carboxyl-terminated PLLA/VMT prepolymers (with 0.5 and $2 \mathrm{wt} \%$ modified-vermiculite respectively) were synthesized by direct polycondensation of L-LA and 0.75 mol\% adipic acid with $\mathrm{SnCl}_{2} \cdot 2 \mathrm{H}_{2} \mathrm{O}(0.5 \mathrm{wt} \%$ based on the mass of L-LA) as catalyst. The synthesis of PLLA-co-bis A ER/VMT was performed by the reaction between carboxylterminated PLLA/ vermiculite prepolymer and bisphenol-A epoxy resin at $180^{\circ} \mathrm{C}$. The molar ratio of bisphenol-A epoxy resin to carboxyl-terminated PLLA/VMT prepolymer was 1.2. All polymerizations were carried out under vacuum [7].

A repeated dispersion/centrifugation process was used to separate the PLLA-grafted VMT nanoparticles (PLLA-gVMT) from the PLLA-co-BisA ER matrix. The PLLA-cobis A ER/VMT nanocomposite was dissolved/dispersed in tetrahydrofuran and then centrifugated at $4000 \mathrm{rpm}$ for $15 \mathrm{~min}$. For the resulting crude VMT nanoparticles, the dispersion/centifugation operation was performed repeatedly for 8-9 times until no absorption of PLLA could be detected by FTIR in the supernatant. After dried under vacuum at $60^{\circ} \mathrm{C}$ for $24 \mathrm{~h}$, the separated PLLA-g-VMT was characterized with TGA and FTIR [8]. 


\subsection{Measurements}

FTIR was performed with Avatar 380 FTIR, Thermo Electron, USA. Transmission Electron Microscope (TEM,JEM-2010) was used to observe the dispersivity of VMT nanoparticles in PLLA matrix. Thermogravimetric Analysis (TGA, NETZSCH-209F1) was performed for the samples of pure PLLA-co-Bis A ER, PLLA-g-VMT, modified VMT and the PLLA-co-Bis A ER/VMT nanocomposites to determine the grafting ratio as well as the thermal stability. The measurement was performed under nitrogen atmosphere and the heating rate was $10{ }^{\circ} \mathrm{C} / \mathrm{min}$. DSC was performed with 204F1, NETZSCH, Germany. The specimens were heated to $190{ }^{\circ} \mathrm{C}$ and maintained at that temperature for 2 minutes to eliminate their thermal history, subsequently they were cooled to $30{ }^{\circ} \mathrm{C}$ at a rate of $80{ }^{\circ} \mathrm{C}$ $/ \mathrm{min}$, and then were heated to $115^{\circ} \mathrm{C}$ at a rate of $80^{\circ} \mathrm{C} / \mathrm{min}$, after stayed at $115^{\circ} \mathrm{C}$ for 30 minutes, they were heated to $190{ }^{\circ} \mathrm{C}$ at a rate of $10{ }^{\circ} \mathrm{C} / \mathrm{min}$. Isothermal crystallization behavior at $115{ }^{\circ} \mathrm{C}$ was observed with an Olympus model BX51 polarized optical microscope.

The viscosity measurements were conducted with a Ubbelohde viscometer with an internal diameter of $0.35 \mathrm{~mm}$; the solvent was THF, and the measurement temperature was $30 \pm 0.1{ }^{\circ} \mathrm{C}$. The weight-average molecular weight of the polymers was calculated by the following formula [9]:

$[\eta]=1.25 \times 10^{-4} M_{w}^{0.717}$

\section{RESULTS AND DISCUSSIONS}

\subsection{The Preparation of PLLA-co-bis A/VMT}

Fourier transform infrared spectroscopy was performed to investigate molecular interactions between the modified vermiculite and PLLA.

Fig. (1) was the FTIR spectra of modified-VMT, Pure PLLA-co-bis A ER and PLLA-g-VMT. In the spectrum of modified-VMT shown in Fig. (1a), the peak at $997 \mathrm{~cm}^{-1}$ and $454 \mathrm{~cm}^{-1}$ can be ascribed to asymmetric stretching vibrations of Si-O-Si and Si-O-Al; The spectrum of PLLA-co-bis A Fig. (1b) exhibits characteristic absorption peaks of easter at $1757 \mathrm{~cm}^{-1}$ for the stretching vibration of $-\mathrm{COO}-$ and at 1060 $\mathrm{cm}^{-1}, 1110 \mathrm{~cm}^{-1}$ and $1191 \mathrm{~cm}^{-1}$ for the stretching vibration of $\mathrm{C}-\mathrm{O}-\mathrm{C}$. The same phenomenon appears in Fig. (1c). The new absorption band at $997 \mathrm{~cm}^{-1}$ and $454 \mathrm{~cm}^{-1}$ can be ascribed to asymmetric stretching vibrations of Si-O-Si and Si-O-Al, and $1626 \mathrm{~cm}^{-1}$ is attributed to -CONH-. These results show that PLLA has been grafted to the surface of vermiculite through the reaction between the carboxyl group of PLLA and the amine group of modified vermiculite surface.

The thermogravimetric analysis (TGA) further provided quantitative evidence of grafting. Fig. (2) shows three typical TGA curves. The pure modified VMT is thermally stable at temperature higher than $700{ }^{\circ} \mathrm{C}$, but the pure PLLA-co-BisA ER thermally degraded at temperature higher than $200{ }^{\circ} \mathrm{C}$. The difference $\left(\mathrm{W}_{1}\right)$ between the residual weight of PLLA-gVMT (sample B) and that of modified VMT (sample A) represents the content of PLLA in PLLA-g-VMT and $\left(1-\mathrm{W}_{1}\right)$ represent the net weight of VMT nanoparticles. So the grafting ratio (Gr) can be calculated using Eq.(2). The grafting ratio is $47.5 \%$.

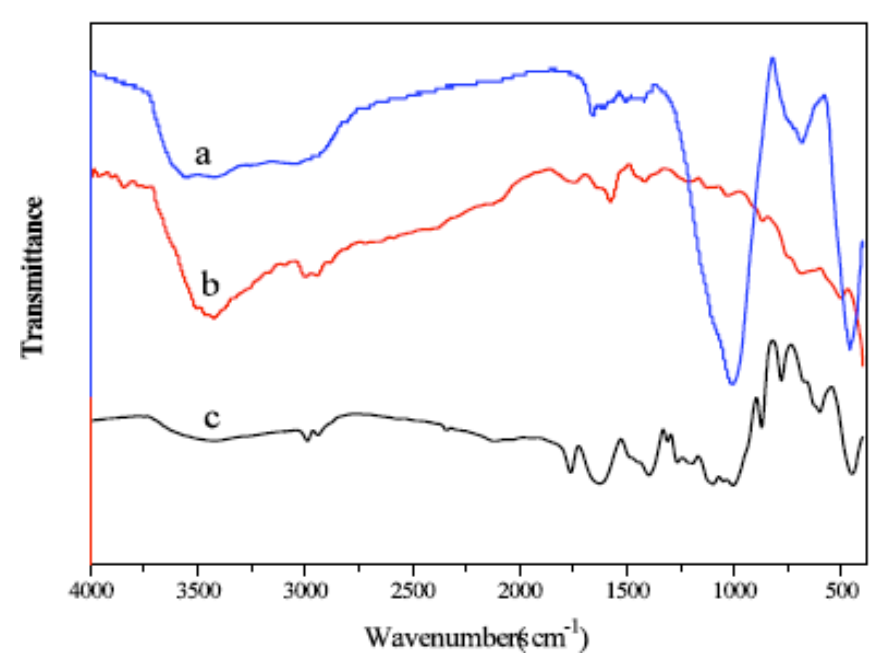

Fig. (1). FTIR spectra of (a) modified-VMT, (b) Pure PLLA-co-bis A ER, (c) PLLA-g-VMT.

$\mathrm{G}_{\mathrm{r}}=\mathrm{W}_{1} /\left(1-\mathrm{W}_{1}\right)$

From the above results, it is concluded that PLLA was chemically grafted onto the surface of the VMT nanoparticles.

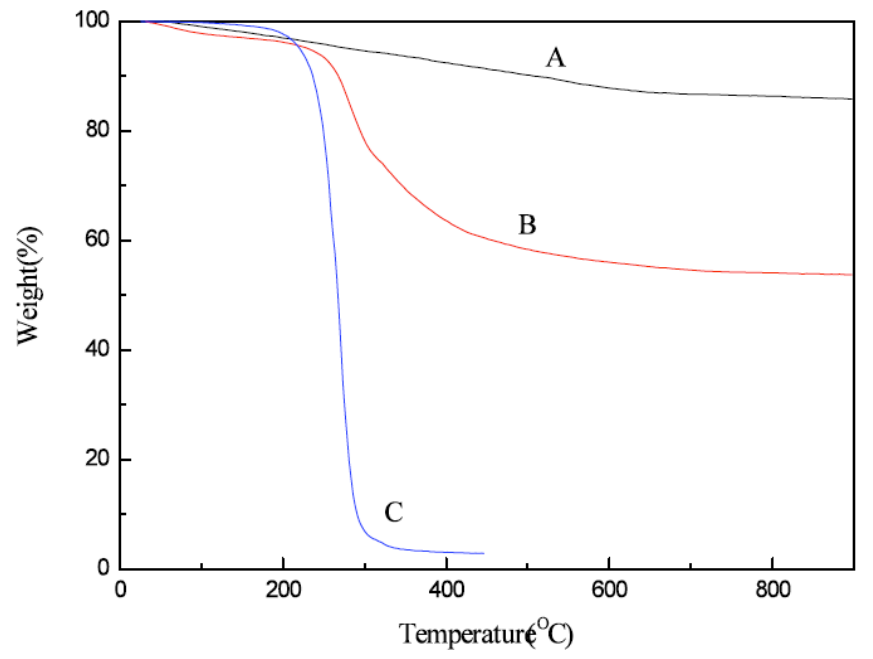

Fig. (2). TGA curves of (A) modified-VMT, (B) PLLA-g-VMT, (C) Pure PLLA-co-BisA ER.

\subsection{Dispersion of VMT Nanoparticles}

Fig. (3) showed the TEM micrographs of PLLA-co-Bis A ER/VMT (2 wt\%) nanocomposite. The light and the dark regions represent PLLA-co-bis A ER and VMT, respectively. From Fig. (3), a heterogeneous disordered nanostructure was observed. The average size of the stacked and exfoliated silicate layers appears to reach about $100 \mathrm{~nm}$ in length, and which are nicely distributed in the PLLA-co-bis A ER matrix. However, we cannot estimate the thickness precisely from the TEM image.

\subsection{Effect of VMT on the Molecular Weight of the Nanocomposites}

The molecular weight of the PLLA-co-bis A ER matrix are important quantities to be concerned. High molecular weight is essential for satisfactory mechanical properties. In many cases the mixing of PLA with clay is followed by a significant drop of the molecular weights, leading to the loss 

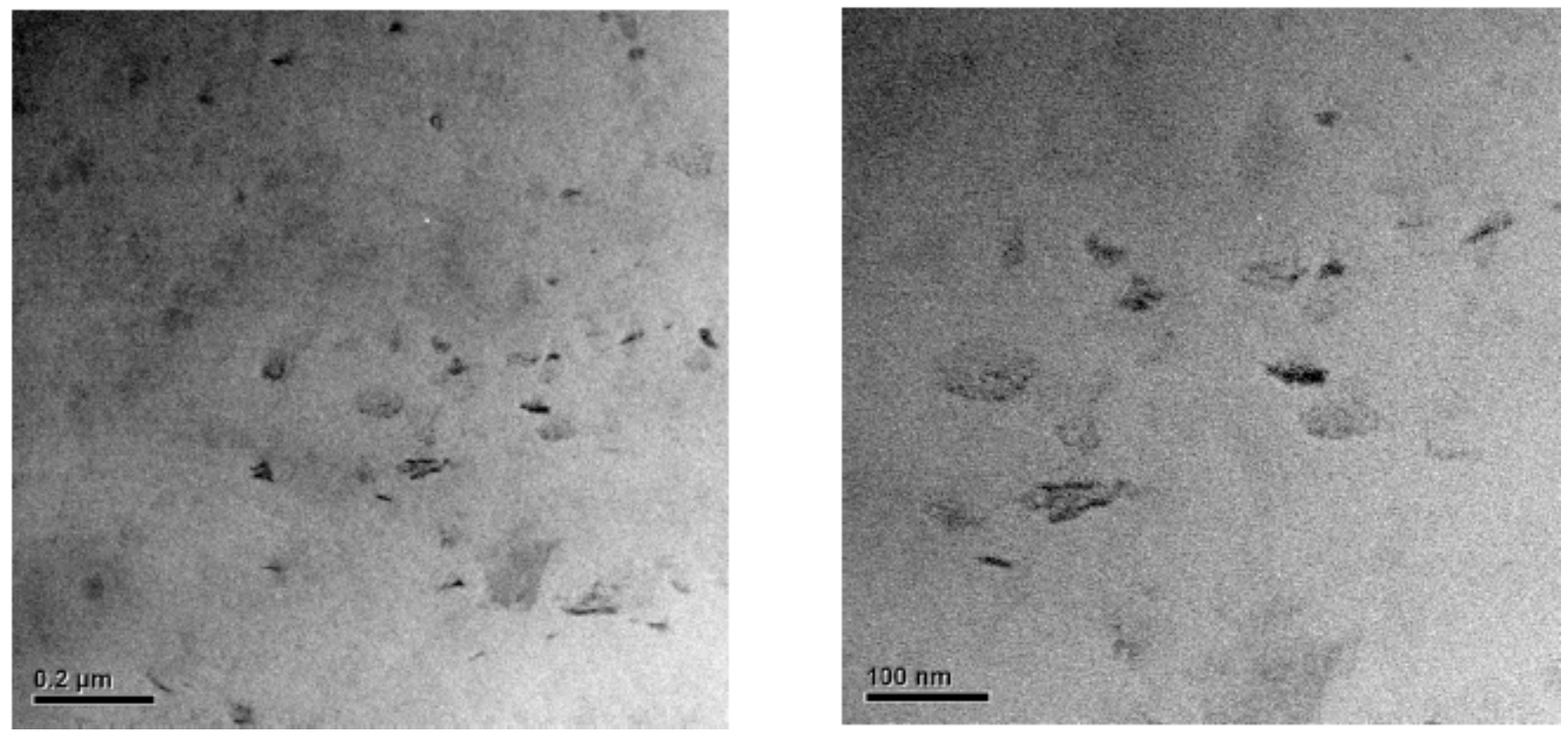

Fig. (3). TEM micrographs of PLLA-co-Bis A ER/VMT nanocomposite with $2 \mathrm{wt} \%$ VMT content.

of the main material properties. The effect of VMT on the molecular weight of PLLA-co-Bis A ER matrix is shown in Table 1.

Table1. Molecular Weight of PLLA/VMT and PLLA-co-Bis A ER/VMT Nanocomposites Prepared by In Situ Melt Polycondensation

\begin{tabular}{|c|c|c|c|}
\hline Run & $\begin{array}{c}\text { Content of } \\
\text { VMT, (WT.\%) }\end{array}$ & $\begin{array}{c}\boldsymbol{M}_{W}, \\
\text { PLLA/VMT }^{\mathbf{b}}\end{array}$ & $\begin{array}{c}\boldsymbol{M}_{W}, \text { PLLA-co-Bis } \\
\text { A ER/VMT }^{\mathbf{c}}\end{array}$ \\
\hline \hline 1 & 0 & 36655 & 145500 \\
\hline 2 & 0.5 & 37602 & 126100 \\
\hline 3 & 1 & 30914 & 124900 \\
\hline 4 & 2 & 30109 & 81800 \\
\hline
\end{tabular}

${ }^{\mathrm{a}} \mathrm{VMT}$ content in the nanocomposite

${ }^{\mathrm{b}}$ Weight-average molecular weight of the PLLA matrix.

${ }^{c}$ Weight-average molecular weight of the PLLA-co-Bis A ER matrix.

From Table 1 it can be found that the molecular weight of PLLA/VMT nanocomposites with $0.5 \mathrm{wt} . \%$ was nearly the same as the pure PLLA. With continuous increasing clay content, the molecular weight of the PLLA/VMT decreased to 30109 when the amount of VMT went up to $2 \mathrm{wt} \%$. In the presence of $0.5 \mathrm{wt} . \% \mathrm{VMT}$, the molecular weight of the PLLA-co-Bis A ER/VMT nanocomposite decreased form 145500 to 126100 , and reached the minimum of 81800 with 2 wt.\% VMT loading.

Two possible reasons are responsible for the reduction of molecular weight. First, the presence of extra amino on the VMT particles broke the intrinsic balance of carboxyl and hydroxyl (COOH/OH 1:1) in LLA, and therefore reduced the theoretical molecular weight at a complete conversion of COOH. Second, at the end of the in situ melt polycondensation, PLLA chains were in high viscosity. It is not easy for chains move freely. When VMT nanoparticles are incorporated into the polymers, the VMT nanoparticles act as barrier. Then the movement of PLLA chains is harder. So the molecular weight of the polymer decreased with the continuous increasing clay content.

\subsection{Thermal Stability}

A representative TGA cruve for the PLLA-co-bis A ER and PLLA-co-bis A ER /VMT 2\% samples is illustrated in Fig. (4). When comparing the onset of thermal degradation for PLLA-co-bis A ER /VMT with PLLA-co-bis A ER, an important increase $\left(13{ }^{\circ} \mathrm{C}\right)$ is observed. This is due to the vermiculite acts as a heat barrier during decomposition, as mentioned [10]. The significantly improvement in the thermal stability of the nannocomposites can also be attributed to strong interfacial interactions between groups on the silicate layer surfaces and the macromolecular chains in the nanocomposites [11].

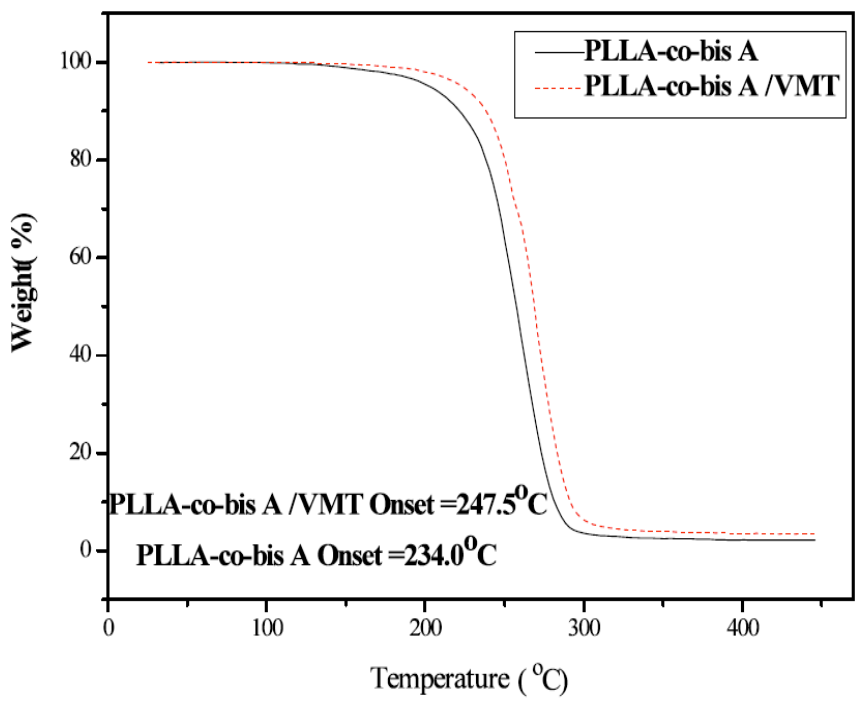

Fig. (4). TGA of PLLA-co-bis A ER and PLLA-co-bis A ER/VMT. 


\subsection{Crystallization Behavior of PLLA-co-bis A ER/Vermiculite Nanocomposites}

The DSC isothermal and second heating thermograms for neat PLLA-co-bis A and PLLA-co-bis A/VMT are presented in Fig. (5). It was found that the crystallization enthalpies during isothermal of the nanocomposites was higher than that of neat PLLA-co-bis A ER. It had been demonstrated that the clay could act as a nucleation agent and the exfoliated layers could play a template role for the crystallization of PLLA-co-bis A ER.

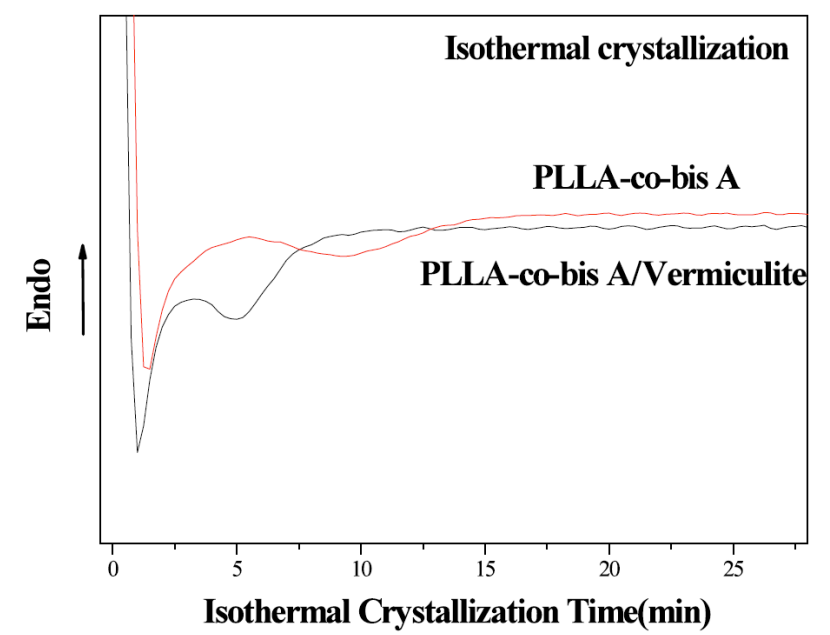

During the second heating scan of neat PLLA-co-bis A ER, two overlapping endothermic melting peaks were presented, and these can be due to the less perfect crystals had enough time to melt and reorganized into crystals with higher structural perfection, which re-melt at higher temperature during the slow DSC scanning.

These results implied that the modified-Vermiculite played a heterogeneous nucleation role, and enhanced the crystallization ability of PLLA-co-bis A ER. However, the addition of clay did not significantly alter the overall crystal structure.

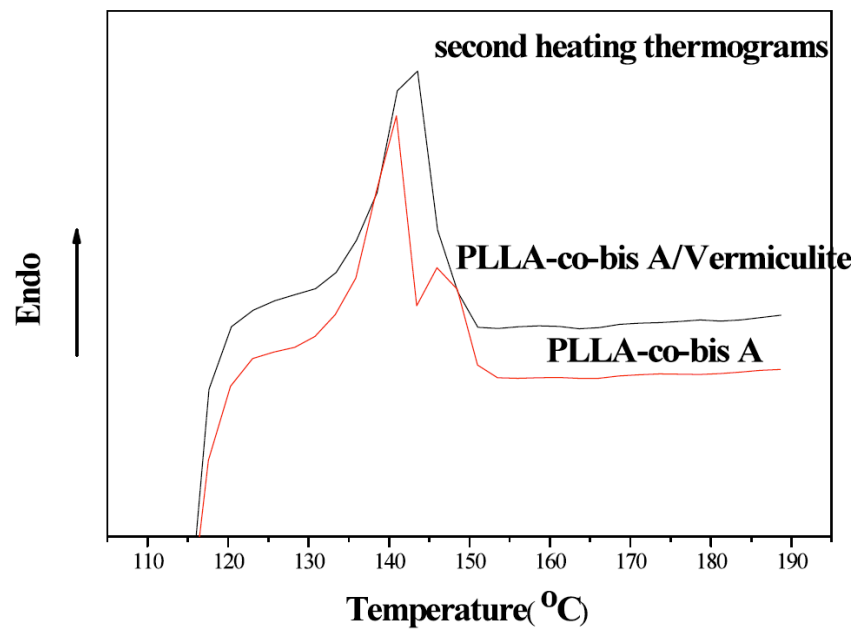

Fig. (5). The DSC curves of cold isothermal crystallization of PLLA-co-bis A ER and PLLA-co-bis A ER/VMT at $115^{\circ} \mathrm{C}$ for $30 \mathrm{~min}$.
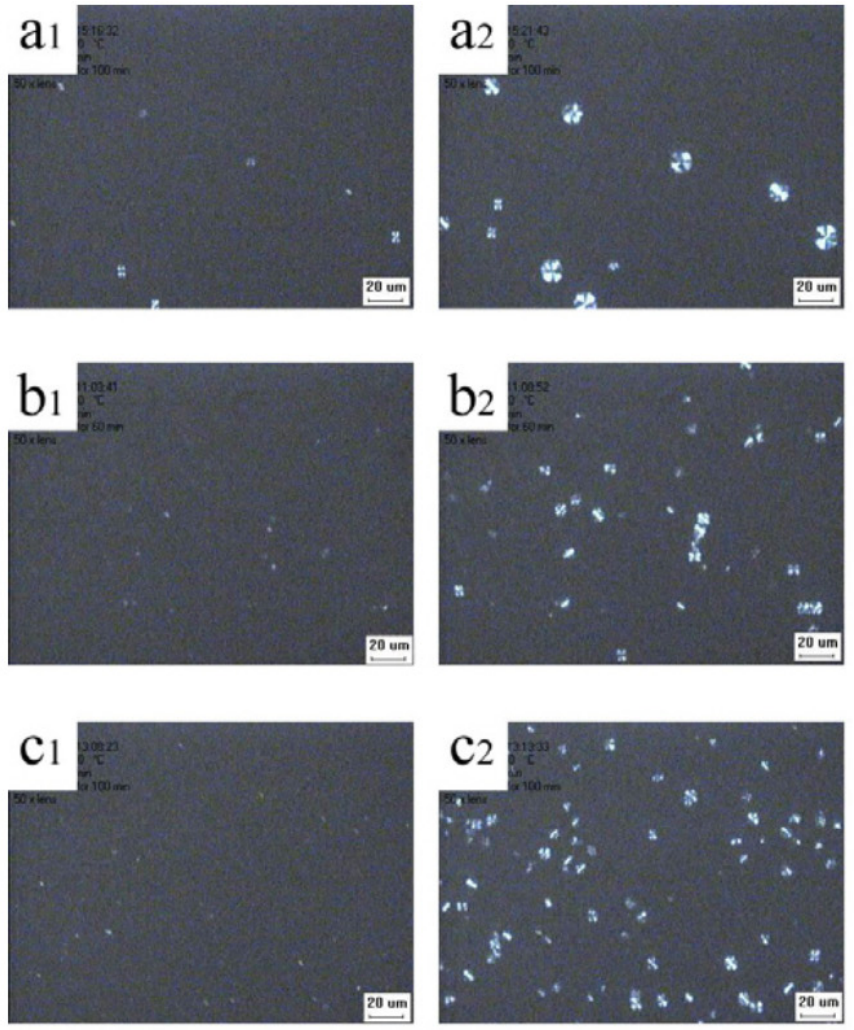

$5 \mathrm{~min}$

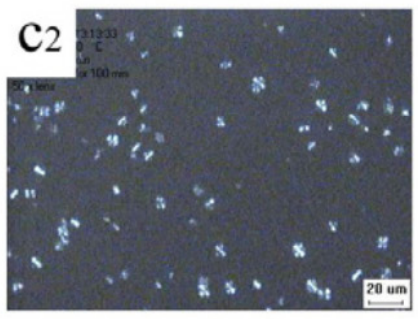

$10 \mathrm{~min}$
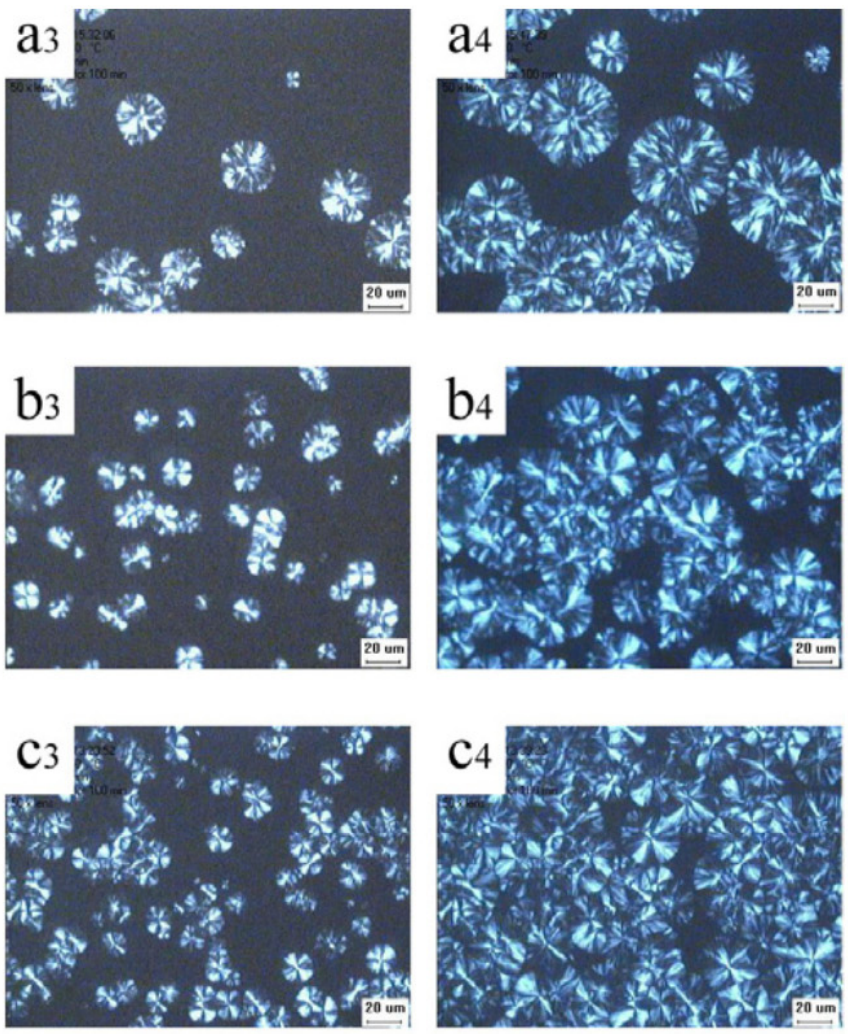

$20 \mathrm{~min}$

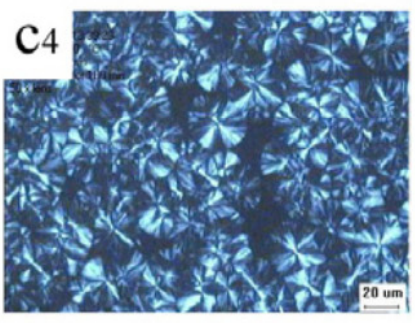

$35 \mathrm{~min}$

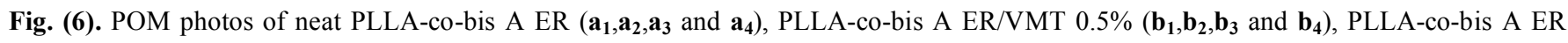
/VMT $2 \%\left(\mathbf{c}_{1}, \mathbf{c}_{2}, \mathbf{c}_{3}\right.$ and $\left.\mathbf{c}_{4}\right)$ crystallized for various times under isothermal heat crystallization condition. 
POM was used to illustrate the difference of the crystal morpholohies between PLLA-co-bis A ER and PLLA-co-bis A ER/VMT nanocomposites. Fig. (6) presents the POM image of PLLA-co-bis A ER, PLLA-co-bis A ER /VMT0.5\% and PLLA-co-bis A ER /VMT2\% crystallized at $115^{\circ} \mathrm{C}$. As shown in Fig. (4), the spherulites were formed in both neat PLLA-co-bis A ER and nanocomposites after isothermal crystallization, and spherulite sizes are systematically increases with the crystallization time. When $0.5 \mathrm{wt} \%$ vermiculite was introduced in PLLA-co-bis A ER matrix, the spherulite became smaller and texture became finer. The result indicated that even a minor weight percentage of vermiculite played a significant nucleation role on the PLLA-co-bis A ER. As the clay content was increased to $2 \mathrm{wt} \%$, the spherulites became dim and showed unclear boundary compared with that of neat PLA indicated that the spherulites were less ordered.

\section{CONCLUSION}

PLLA-co-bis A ER/Vermiculite nanocomposites with high thermal stability and fast crystallization rate were prepared by in situ melt polycondensation. In the resulting nanocomposites, the VMT nanoparticles were chemically grafted with PLLA chains and well dispered on nano-scale in the PLLA-co-Bis A ER Matrix. The grafting ratio reached $47.5 \%$. The vermiculite nanoparticles in the matrix played a heterogeneous nucleation role, enhanced the thermal stability and crystallization ability of PLLA-co-bis A ER.

\section{ACKNOWLEDGEMENTS}

This work was supported by grants from the National High Technology Research and Development Program of China (863 Program) (No. 2007AA03Z336), National Natural Science Foundation of China (Grant No. 21074021), Shanghai Municipal Natural Science Foundation (No.
09ZR1401600) and the Program for New Century Excellent Talents in University (No. NCET-07-0174).

\section{REFERENCES}

[1] Li B, Dong FX, Wang XL et al. Organically modified rectorite toughened poly(lactic acid): Nanostructures, crystallization and mechanical properties. Eur Polym J 2009; 45: 2996-3003.

[2] Drumright RE, Gruber PR, Henton DE. Polylactic acid technology. Adv Mater 2000; 12: 1841-6.

[3] Nam JY, Sinha Ray S, Okamoto M. Crystallization behavior and morphology of biodegradable polylactide/layered silicate nanocomposite. Macromolecules 2003; 36:7126 -31.

[4] Paul MA, Alexandre M, Degee P, Calberg C, Jerome R, Drbois P. Exfoliated polylactide/clay nanocomposites by in situ coordinationinsertion polymerization. Macromol Rapid Commun 2003; 24: 561-6.

[5] Ogata N, Jimenez G, Kawai H, Ogihara T. Structure and thermal/mechanical properties of poly(L-lactide)-clay blend. Polym Sci B, Polym Phys 1997; 35: 389-96.

[6] Zang L, Luo J, Guo J, Liu H, Ru J. Preparation and characterization of poly(ethylene glycol) /organo-vermiculite nanocomposite polymer electrolytes. Polym Bull 2010; 65(7): 669-80.

[7] Xu H, Teng CQ, Yu MH. Improvement of thermal property and crystallization behavior of PLLA based multiblock copolymer by forming stereocomplex with PDLA oligomer. Polymer 2006; 47: 3922-28.

[8] Wu L, Cao D, Huang Y, Li B. Poly(l-lactic acid)/SiO $\mathrm{S}_{2}$ nanocomposites via situ melt polycon-densation of 1-lactic acid in the presence of acidic silica sol: Preparation and Characterization. Polymer 2008; 49: 742-8.

[9] Kricheldorf HR, Lee SR, Bush S. Polylactones 36. Macrocyclic polymerization of lactides with cyclic $\mathrm{Bu}_{2} \mathrm{Sn}$ initiators derived from 1,2-ethanediol, 2-mercaptoethanol, and 1,2-dimerc-aptoethane. Macromolecules 1996; 29:1375-81.

[10] Sinha Ray S and Okamoto M. Polymer/layered silicate nanocomposites: A review from preparation to processing. Prog Polym Sci 2003; 28: 1539-1641.

[11] Jin FL, Rhee KY and Park SJ. Surface treatment of montmorillonite on the thermal stabilities of bisphenol-A diglycidyl dimethacrylate nanocomposites. Mater Sci Eng A-Struct 2006; 435: 429-33. 